\title{
La educación con sentido comunitario: reflexiones en torno a la formación del profesorado
}

\author{
ALMA ARCELIA RAMÍREZ IÑIGUEZ* \\ Cátedras CONACYT - México \\ Programa Interdisciplinario sobre Política y Prácticas Educativas (PIPE-CIDE) \\ Recibido el 06-03-2016; primera evaluación el 14-02-2017; \\ segunda evaluación el 02-05-2017; aceptado el 10-06-2017
}

\section{RESUMEN}

Este artículo presenta un análisis sobre las posibilidades de formar al profesorado de educación básica en relación con el contexto en el que desarrolla su labor profesional. Para ello, se parte de una revisión conceptual sobre el vínculo entre educación y comunidad con la finalidad de explicar las características de los procesos formativos que se llevan a cabo desde una visión comunitaria, así como sus implicaciones. A partir de este análisis se establecen algunas orientaciones sobre los aspectos a considerar en la formación del profesorado en este sentido, destacando una visión sistémica de la educación, dentro de la cual los factores escolares y los del entorno se relacionan, y en la que el profesor es un agente en continuo aprendizaje.

Palabras clave: educación básica, relación escuela comunidad, formación docente.

\section{Community-based education: reflections on teacher education}

\section{Abstract}

This article presents an analysis about teacher's education possibilities regarding their professional context in elementary education. For this, a conceptual review about relationship between education and community is explained. In this analysis, the educational processes' characteristics from this perspective are highlighted, as well as their implications. Subsequently, some guidelines about teacher's education

\footnotetext{
Doctora en Educación por la Universidad de Barcelona y licenciada en Pedagogía por la Universidad Nacional Autónoma de México. Su desempeño profesional y en investigación se ha centrado en la evaluación educativa, la educación inclusiva e intercultural y la inclusión social. Actualmente, se enfoca al estudio de los factores que propician la inclusión desde el ámbito educativo, especialmente en contextos de vulnerabilidad social. Contacto: almarceliar@gmail.com
} 
from this view are exposed and a systemic approach of education are presented taking into account educational factors inside and outside the school, as well as the teacher as an agent who learns permanently.

Keywords: elementary education, school-community relations, teacher education.

A sensação de educaçáo comunitária: reflexões sobre a formação de professores

\section{RESUMO}

Este artigo apresenta uma análise sobre as possibilidades de formar professores de educação básica relacionado com o contexto em que desenvolvem seu trabalho profissional. Para tanto se partirá de uma revisão conceitual sobre o vínculo entre a educação e a comunidade, com o objetivo de explicar as características dos processos formativos que desenvolvem dentro de uma visão comunitária, assim como suas implicaçôes. A partir desta análise se estabelecem algumas orientaçôes sobre os aspectos a serem considerados na formaçáo do professorado, enfatizando uma visáo sistêmica da educação, dentro da qual a escola e o meio ambiente estáo inter-relacionados, e na qual o professor é um agente em contínua aprendizagem.

Palavras-chave: educação básica, relação escola comunidade, formação docente.

\section{LA COMUNIDAD COMO REFERENCIA EN LOS PROCESOS EDUCATIVOS}

Las acciones educativas plantean determinados propósitos de acuerdo con el desarrollo deseado en los individuos y en su entorno. En este sentido, los proyectos de formación establecen ciertos conocimientos, habilidades y actitudes que se espera lograr en las personas, tomando en cuenta su interacción con los demás. Por lo tanto las expectativas en los procesos educativos orientarán sobre aquello que es deseable obtener como resultado de diversas acciones de aprendizaje personal y colectivo. Posicionar dichos procesos teniendo como referencia a la comunidad exige acotar su definición.

Un concepto ampliamente extendido es el que se refiere a la idea de un territorio y de una identidad compartida (Johansen $\&$ Nielsen, 2012) a partir de la cual se determina el grado de cohesión que tienen diversos grupos en un marco de espacio-tiempo. Sin embargo, más allá del ámbito territorial, el concepto de comunidad remite al establecimiento de vínculos de proximidad y emotividad, en los que además de interdependencia entre los individuos, existe un consenso internalizado de acercamiento que rebasa la agregación (Subirats, 2003). De esta manera, una parte de la subjetividad personal se comparte con la colectiva al definir valores, creencias o intereses mutuos. Por lo tanto, el sentido comunitario implica un tejido de conocimientos, actitudes y habilidades que se consideran necesarios para la socialización (Lavaniegos, 2008). 
Además, la comunidad plantea relaciones entre los individuos en las que existen procesos de empoderamiento, participación y satisfacción recíprocas (Ramos, Holgado \& Maya, 2014), en tanto que las personas tienen un papel activo en los vínculos que establecen y su desarrollo se retroalimenta de las interacciones producidas. De este modo, la comunidad tiene un sentido de ayuda mutua y responsabilidad en tanto que la relación entre las personas no es estéril sino que se construye hacia un bienestar común, es decir, todos los sujetos se reconocen y se enriquecen con los vínculos.

Por otro lado, la comunidad se distingue por su pluralidad, es decir, existen múltiples formas de crear comunidad. Por una parte, se ubica como un territorio o localidad en donde las personas interactúan y comparten de manera presencial. Por otra, la comunidad es entendida en su dimensión relacional, es decir, se crea a partir de los intereses de los sujetos con independencia de su lugar geográfico (Subirats, 2003; Maya, 2004). Así, tomando en cuenta que las principales características de lo comunitario se relacionan con lo personal, lo relacional y lo afectivo, la proximidad entre las personas es la que define la existencia de una comunidad. En este sentido, se destaca que su construcción depende más de los vínculos que se establecen a nivel subjetivo entre los sujetos que de los espacios físicos en los que se desenvuelven.

De hecho, algunos autores (Maya, 2004; Rodríguez, 2006; Jaramillo, 2007) destacan que en el escenario de la globalización actual, los límites de lo que significa construir una comunidad se vuelven porosos, pudiendo existir sentidos de pertenencia sin compartir necesariamente espacios físicos. Cualquiera que sea su modalidad, una comunidad tendrá como referencia conceptual aquellos espacios locales o virtuales en donde las personas establecen lazos de reconocimiento, solidaridad y ayuda mutua con base en una diversidad de identificaciones y afinidades que fomentan correspondencias, generando bienestar individual y social.

Por lo tanto, los procesos educativos que tienen como punto de referencia a la comunidad centran sus propósitos en el desarrollo de todos los grupos sociales que conforman un colectivo. De este modo, no se trata de procesos individualistas orientados únicamente a la mejora personal sino que la educación cobra sentido cuando se lleva a cabo en dinámicas compartidas donde todos tienen algo para enseńar y aprender.

Desde este enfoque, la educación tiene como propósito dar respuesta a las demandas sociales (Cieza, 2006), las cuales parten de requerimientos individuales pero en relación con las necesidades de los otros. En consecuencia, las personas se forman en un contexto dentro del cual el bienestar individual es importante en tanto que impacta en el bienestar de los demás. La escuela, 
en este sentido, es vista como una comunidad en la que todos se comprometen y trabajan conjuntamente para mejorarla (Gallego, Rodríguez \& Corujo, 2016), por lo que tanto profesores como familias y diferentes agentes sociales se corresponsabilizan de los resultados educativos que se obtienen.

Así, desde la perspectiva comunitaria, las acciones educativas pueden ser caracterizadas de la siguiente manera (Bueno, 1991; Gallego, 2010; Bustos, 2011):

- Son procesos participativos en los que el profesorado, el alumnado, las familias y diversos agentes sociales se involucran en las decisiones que se toman en torno a la escolarización y la educación fuera del ámbito escolar.

- Atienden los intereses locales retomando sus características lingüísticas, culturales y de vida social, de tal manera que los procesos educativos se vinculan con el devenir de la comunidad.

- Se buscan experiencias que favorezcan una participación en condiciones de igualdad entre los agentes escolares y no escolares implicados, a través del reconocimiento del desarrollo social en su conjunto y de las garantías de todos los involucrados.

- Se orientan hacia la transformación de las condiciones que impiden el desenvolvimiento de todas las personas dentro de la comunidad mediante la construcción de lazos entre distintos profesionales, agentes y acciones que buscan lograr, desde sus ámbitos de conocimiento, objetivos comunes.

Bajo estos preceptos, la educación es un proceso dirigido al desarrollo de los sujetos más allá de la competitividad y la satisfacción personal, en el cual la comunidad es un aspecto importante dentro de iniciativas que consideran el entorno como parte fundamental en la adquisición de los aprendizajes básicos para vivir y convivir. Cabe señalar que, desde este enfoque, la comunidad no solo es un recurso al cual se hace referencia para favorecer los aprendizajes escolares, sino que es el marco dentro del cual se llevan a cabo estos aprendizajes, así como diversas acciones que tienen la finalidad de propiciar la adquisición de competencias básicas para el desarrollo social. Por lo tanto, la educación no solo es un beneficio para las personas en tanto que posibilita su crecimiento, sino que constituye procesos que favorecen a toda la comunidad a partir de la atención de sus individuos y el despliegue de sus capacidades para el beneficio social.

Los análisis de algunas experiencias realizadas bajo este planteamiento (Cieza, 2006; Morińa \& Parrilla, 2006; Bustos, 2011; Castro, Rodríguez \& Urteaga, 2013) explican cómo los procesos educativos comunitarios impactan socialmente. Una de sus características más sobresalientes es su relevancia 
para contrarrestar la segregación y la marginación. En estas, se busca que los conocimientos y los códigos de los grupos históricamente desposeídos, socioculturalmente desfavorecidos o empobrecidos obtengan un reconocimiento en el devenir cotidiano de la sociedad. De esta manera, son tomadas en cuenta sus capacidades para intervenir en las decisiones que les afectan, y en el diseño de las acciones que les permiten la modificación de aquellos elementos del entorno que los mantienen en condiciones de desigualdad. Específicamente, en la experiencia analizada por Cieza (2006) se reconoce que el desarrollo comunitario es cada vez más una aspiración de cualquier proyecto social, político, económico o educativo con una base democrática que aspira al bienestar de todas las personas reduciendo las desigualdades y atendiendo sus necesidades básicas. En la revisión de dichas experiencias educativas se destacan los siguientes aspectos.

- La realidad como base para la problematización. Se trata de proyectos en los que se resignifican los textos, los contenidos y su organización a partir de los sucesos e intereses locales, así como de los problemas más urgentes. Por lo tanto, la reflexión es una constante en la planeación y puesta en práctica de las propuestas educativas, las cuales se vinculan con los acontecimientos cotidianos.

- Flexibilidad como principio. Para realizar el punto anterior, los proyectos educativos son dinámicos, están en continuo cambio y se basan en la reflexión continua, de tal manera que se posibilita la adecuación de los mismos a las demandas sociales. En este sentido, los recursos disponibles son utilizados de manera eficiente, adaptándose a las necesidades que van surgiendo. Igualmente se les da una importancia fundamental, al ser básicos para la puesta en marcha de las propuestas educativas.

- Participación como eje principal. Todas las personas implicadas en los procesos de formación (madres, padres, profesorado, alumnado, agentes externos a la escuela) colaboran e inciden en la toma de decisiones, así como en las normas para el funcionamiento de los acuerdos establecidos. De esta manera, el profesorado trabaja conjuntamente en la elaboración de propuestas y resolución de problemas; las diferentes entidades sociales facilitan el trabajo docente; el alumnado y sus familias aportan sus conocimientos, vivencias y apoyo en el desarrollo del proyecto, y sus sugerencias son tomadas en cuenta. Por lo tanto, el profesorado conoce la cultura de la escuela y la de la comunidad. Así, la participación constituye una fuente de aprendizaje para todos.

- Negociación como ejercicio cotidiano. Parte de este aprendizaje se basa en el contraste de ideas a partir de la escucha y del intercambio de 
experiencias de todos los participantes, lo cual propicia el análisis de los diferentes elementos que conforman el proyecto educativo y la colaboración para su realización, así como para la resolución de problemas. Además, la heterogeneidad dada por los diferentes grupos involucrados fomenta el reconocimiento de todos y la interdependencia más que la subordinación de unos grupos a otros.

- Ayuda mutua. Como consecuencia de los puntos anteriores, los colectivos comunitarios organizan las acciones pertinentes para alcanzar los objetivos educativos propuestos. De este modo, se gestionan los procesos que le permiten al profesorado llevar a cabo su labor, se proporciona ayuda a las familias para que contribuyan en el desarrollo de la propuesta educativa, y se establecen conjuntamente las estrategias y los dispositivos que propician el aprendizaje de todo el alumnado.

- Profesorado como agente de transformación. La función docente rebasa las formas tradicionales de enseñanza y se abre a los espacios fuera de las aulas. De esta manera, el profesorado se concibe como un agente no solo de enseńanza sino también de aprendizaje trabajando de manera colegiada, intercambiando ideas y propuestas con madres, padres y otros agentes de la comunidad para lograr propósitos de formación comunes dentro de una relación de reciprocidad.

Ubicar los procesos educativos en el contexto comunitario significa la posibilidad de crear prácticas formativas que favorecen el desarrollo de todos los integrantes de una sociedad. Por ello, se trata del establecimiento de estrategias que rebasan el espacio escolar y que implican una participación conjunta de los múltiples actores que conforman el entorno social. La importancia de dicha participación radica en la incidencia de los agentes escolares y sociales para la construcción de dinámicas donde el alumnado en su totalidad es tomado en cuenta y en donde se generan vínculos que retroalimentan el desarrollo de sus competencias.

En estos procesos se crean ámbitos de comunicación multidireccional en donde todos aprenden de los conocimientos y experiencias del resto. Por lo tanto, se abren canales de reciprocidad y compromiso para alcanzar metas en común, especialmente aquellas referidas a transformar las condiciones que crean exclusión.

De esta manera, la comunidad, como el espacio en el cual se suscitan diversidad de relaciones, se convierte en el ámbito que posibilita la mejora de las formas de vida de los sujetos. Al respecto, los procesos educativos no son prácticas aisladas de los acontecimientos sociales sino que se concretan en acciones enmarcadas en la vida diaria de la sociedad, teniendo como finalidad contribuir a que los acontecimientos cotidianos beneficien a todos y no solo a unos cuantos. 


\section{COMUNIDAD Y ESCUELA: UNA RELACIÓN SISTÉMICA}

La alusión a la comunidad como parte de la educación implica la consideración de que la escuela no es un agente independiente del resto de organizaciones sociales que de manera explícita o implícita inciden en la formación de las personas. De hecho, desde una visión comunitaria, los procesos educativos se llevan a cabo a través de la articulación de los esfuerzos que se realizan desde otros organismos sociales. En este sentido, este enfoque plantea una relación sistémica entre las acciones educativas en los ámbitos escolares y desde otras agencias. Con base en esta idea se promueven políticas transversales que afectan al conjunto de los componentes de un sistema educativo como son el currículo, la formación del profesorado, la dirección escolar o la financiación (Escudero \& Martínez, 2011), destacándose que la escuela necesita de otras agencias para su funcionamiento (Neirotti, 2008).

De este modo, en la relación sistémica que se establece entre la escuela y los agentes comunitarios se reafirma la necesidad de una amplia participación de diversos actores como los gubernamentales, el mundo académico y la sociedad en general, especialmente de los grupos vulnerables o excluidos (Blanco \& Duk, 2011). Cabe destacar que dicha relación es compleja y requiere del establecimiento de convenios, los cuales son revisados periódicamente para atender las necesidades de la comunidad y de todas personas que la componen. Por lo tanto, se trata de una relación orientada a mejorar las dinámicas que condicionan el desarrollo de los individuos y de su entorno.

En esta relación sistémica, la escuela es concebida como una entidad en la que sus elementos internos están vinculados entre sí y con el entorno, de tal manera que las dinámicas del medio influyen en los procesos educativos y, por lo tanto, en el aprendizaje del alumnado y del resto de los agentes escolares (Bertalanffy, 1981; Hidalgo, Siu, Bright, Swap \& Epstein, 1995). Es así que las características de los proyectos enfocados desde la visión comunitaria se concretan mediante el trabajo colaborativo dentro y fuera del centro escolar, y en el establecimiento de lazos de cooperación y entendimiento. La escuela es el lugar en donde se suscita el intercambio de ideas, experiencias y estímulos, y en donde convergen los esfuerzos de los docentes, el alumnado y sus familias, así como de otros actores.

A través de esta relación se generan acciones colectivas (Bustos, 2011), es decir, se construyen dentro de la escuela procesos educativos basados en las características del alumnado, en donde los docentes reflexionan sobre sus creencias y valores, y en donde las aportaciones de las familias y de la comunidad son consideradas como fuente de conocimiento para la toma de decisiones. 
Por su parte, fuera del ámbito escolar, se crean redes de cooperación para el logro de los objetivos establecidos. Las dinámicas que se generan revelan que relacionarse de manera colaborativa es una pieza clave en las actividades escolares y extra escolares (Bustos, 2011). En ambas se abren posibilidades para trabajar con el barrio (Castro et al., 2013) en una educación dialógica centrada en las necesidades de las personas y de la sociedad (Carneiro, 2012).

De este modo, se remite a una relación que fomenta el compromiso (Perrenoud, 2005). El grado de responsabilidad que se asume es el resultado del análisis continuo de los sucesos educativos y sociales, los acuerdos que se van estableciendo, así como la coordinación y la realización de las estrategias que se consideren más apropiadas para alcanzar los propósitos planteados. Por ello, un aspecto que define a este tipo de relación es la colaboración entre la escuela, las familias y la comunidad. Para lograrla, es necesario reconocer las fortalezas del centro y las barreras que dificultan la participación de todos los implicados en el proceso educativo, tener voluntad para escuchar las diferentes necesidades, analizar las formas en las que se está contribuyendo al logro educativo de todo el alumnado y poner en práctica aquellos cambios que se consideren pertinentes (Simón, Giné \& Echeita, 2016).

Así, la escuela en su relación de correspondencia con la sociedad no es un ente desde que el cual se mira de forma pasiva lo que acontece en el entorno sino que el devenir comunitario se incorpora a la vida escolar. Al existir negociaciones y roles de compromiso, ninguno de los participantes percibe a los otros como invasores de sus espacios de trabajo sino como actores que contribuyen a la mejora del mismo. Tanto el profesorado como los directivos, el alumnado, las familias y las organizaciones sociales entienden que las intervenciones que realiza cada participante favorecen el logro de fines comunes. De esta manera, dentro de la relación sistémica de la comunidad con la escuela, todos los participantes se mantienen en una postura de aprendizaje, flexibilizando sus perspectivas para facilitar la comprensión y el trabajo integrado.

De manera específica, el profesorado dentro de un enfoque comunitario de la educación, se convierte en un actor que se posiciona no solo como enseñante sino también como aprendiz. Rebasa el rol tradicional de poseedor de los conocimientos que merecen ser transmitidos, y se asume como un agente que aprende de sus estudiantes y de los actores que tienen una incidencia en los procesos educativos. Dentro de los modelos que reconocen el papel de los agentes del entorno en la educación escolar, el profesorado es un actor central en las escuelas que buscan la transformación en esta dirección (Cardona \& Ortells, 2012). La actitud con la que los docentes se desempeñan como educadores resulta fundamental para promover los aprendizajes y para asumirse como parte de un contexto social 
determinado. Por lo tanto, sus funciones están correlacionadas con las acciones comunitarias, de tal manera que incorporan ideas, conocimientos y estrategias en el trabajo colaborativo con el resto de integrantes escolares y sociales.

En el caso de los docentes de educación básica, la relación que establecen con las familias resulta ser uno de los aspectos clave para la integración de esfuerzos y actuaciones dentro y fuera del ámbito escolar. El trabajo en conjunto propicia una conexión en la que todas las partes se reconocen como corresponsables de la educación. En esta correspondencia existe una sensibilidad hacia las características culturales, raciales y sociales de los participantes (Chieh \& Vázquez, 2009), de tal manera que las personas se sienten parte de los procesos de desarrollo educativo y social. En este sentido, las contribuciones que se generan como resultado de este vínculo son consideradas dentro de un esquema de respeto hacia la diferencia, tomando en cuenta que en las dinámicas de participación y en el establecimiento de acuerdos, el desarrollo de las personas para el bien común es el eje en el que se fundamentan las decisiones. Así, es necesaria la re-conceptualización del trabajo docente (Meza, 2011), el cual no se circunscribe al aula sino que está en relación con el ámbito social y contribuye a la construcción de un entorno responsable, participativo y armónico.

Desde este marco conceptual, la educación tiene una función social y los docentes son un importante agente para la transformación de las condiciones que limitan el desarrollo de las personas. Por ello, al ejercer su función de enseñanza también se conciben como agentes de aprendizaje, puesto que la planeación y la conducción de sus actividades, así como la reflexión sobre su práctica se realizan en relación con el resto de los actores comunitarios. De esta manera, los docentes constituyen un elemento fundamental del sistema, coadyuvando al bienestar de las personas y de la comunidad mediante la educación. En el ejercicio de sus competencias, el profesorado asume las influencias del entorno como desafíos que estimulan su trabajo, con la finalidad de adaptarse a las circunstancias en las que se desempeña, y a partir de las cuales se perfecciona para responder a las necesidades de su alumnado.

Así, desde una perspectiva comunitaria, su formación y desenvolvimiento son parte del conglomerado de acciones que lo preparan para adaptarse, contribuir al desarrollo de la sociedad y de sus miembros, e innovar sus actividades profesionales teniendo como referencia las características del contexto. En este sentido, el profesorado no adquiere determinados conocimientos y habilidades para cumplir con los objetivos de un programa educativo que le es ajeno, sino que se siente parte de un proyecto que lo acoge y en el que se involucra junto con el resto de las personas que lo constituyen. De este modo, su formación no se establece sobre la base de conocimientos estáticos en los que se instruye 
para posteriormente impartirlos, sino que se fundamenta en el aprendizaje de contenidos y principios que le posibilitan, a su vez, la creación de espacios dentro de los cuales las personas adquieren las competencias básicas para vivir y transformar su medio de acuerdo con sus necesidades y con las de los demás.

\section{EL DESEMPEÑO DOCENTE: UNA FORMACIÓN EN CONTEXTO}

A partir de lo expuesto anteriormente, se reafirma que, desde un enfoque comunitario, el profesorado es un agente abierto que evalúa sus acciones con base en los vínculos que establece al interior de las aulas, fuera de ellas, en los centros educativos y con las organizaciones sociales vinculadas con su labor. Por lo tanto, se destaca que su formación debe ser continua, contextualizada y fundamentada en la reflexión sobre el propio desempeño. De esta manera, su mejora profesional se circunscribe en un proceso de continuo análisis, no solo por parte de agentes externos a su labor, sino con base en la autocrítica.

$\mathrm{Al}$ plantearse una relación sistémica tanto al interior de las escuelas como con los agentes del entorno, los docentes participan en las decisiones que impactan su actividad profesional. En este sentido, desde su formación inicial, el vínculo que se crea con la comunidad proporciona una dirección al desarrollo de las competencias requeridas para llevar a cabo sus funciones, relación en la cual se consideran sus puntos de vista y aportaciones. Así, los procesos de capacitación docente responden a las necesidades y a las preocupaciones del profesorado (Morińa y Parrilla, 2006), el cual, a su vez, se vincula con los intereses de la comunidad reflejados en su alumnado, en la relación que establece con las familias y en su participación dentro de los proyectos que delimitan el marco de su desempeño.

Por otro lado, la comunidad, como el marco en el cual el profesorado lleva a cabo su labor, conforma el principal referente para su capacitación formal y para los procesos de reflexión sobre la propia práctica. De esta manera, partiendo de la formación inicial hasta los proyectos de profesionalización permanente, la heterogeneidad de los grupos de capacitación, así como la interdisciplinariedad de los mismos fomentan la aceptación de la diversidad, la flexibilidad del pensamiento y el trabajo colectivo (Morińa \& Parrilla, 2006; Bustos, 2011; Castro et al., 2013). Por lo tanto, dentro de los espacios de formación del profesorado, deben incentivarse las capacidades que le posibilita una intervención educativa orientada al desarrollo de individuo y de la colectividad, vinculándose con las demandas sociales y atendiendo las necesidades de las personas en relación con su medio.

Algunas experiencias en este sentido (Meza, 2011) indican que los docentes comparten sus conocimientos y vivencias con otros miembros de su entorno 
y con las instituciones comunitarias. Asimismo, se movilizan dentro de sus centros escolares para atender las problemáticas detectadas y reflexionan sobre estas, proponiendo acciones para su atención. Desde su formación inicial hasta la que realizan durante su trayectoria profesional, los docentes concientizan que su labor no se acota a las aulas ni al logro de aprendizajes en términos exclusivamente de rendimiento. Se dan cuenta del impacto que tienen en el desarrollo presente y futuro de su alumnado dentro de la sociedad, por lo que trabajan no solamente para el individuo, sino que su desempeño va más allá del ámbito escolar.

Desde una visión comunitaria, el profesorado de educación básica impulsa los aprendizajes de competencias esenciales para que las personas se desarrollen en su comunidad con base en su propio aprendizaje. Por ello, una formación del profesorado contextualizada implica romper las formas tradicionales de preparación profesional (Castro et al., 2013) en donde la capacitación está enfocada a la transmisión y actualización de conocimientos. Por el contrario, se trata de procesos formativos en los que la práctica y su análisis son una constante. Cabe señalar que desde esta perspectiva de la educación, la escolarización básica tiene como propósito el desarrollo de las competencias que les permiten a las personas vivir y compartir con quienes conviven, formándolos con los conocimientos, las habilidades y las actitudes necesarias para modificar aquellos aspectos que limitan su desarrollo y el de las personas de su entorno.

Así, se piensa en una educación básica que empodera a los sujetos para el ejercicio de sus derechos (Gutmann, 2004) y para su completa participación dentro de sus sociedades (Unesco, 2005). Es en esta dirección hacia la que el ejercicio docente apunta y en la que la relación con los agentes del entorno contribuye. Por lo tanto, la formación del profesorado, desde esta perspectiva, se basa en los mismos principios educativos a los que se desea dirigir su trabajo profesional. La formación de los docentes, en este sentido, no se acota a una serie de lineamientos que deben seguir de manera mecánica, sino que se basa en la interiorización de valores que se adquieren desde una formación práctica y reflexiva, la cual es acompañada con un asesoramiento desde y para la comunidad basado en la solidaridad y el compromiso mutuo, en la toma de decisiones de forma participativa y en la promoción de una formación situada a partir de la cual se transforman los escenarios educativos en realidades concretas (Rodríguez, 2006). Por lo tanto, se parte de una formación que se espera replicar en la labor de enseñanza. Se trata de una preparación permanente que no termina en la formación inicial sino que forma parte de los retos a los que continuamente el profesorado se va enfrentando de acuerdo con los cambios que va teniendo la propia comunidad y, por lo tanto, su propia práctica. 
De esta manera, su formación se caracteriza por tener como finalidad un desempeńo vinculado con el conjunto de elementos que influyen en su labor y que contribuyen al logro educativo de su alumnado. En este sentido, se trata de una visión inclusiva de la educación y, como tal, su formación se contextualiza en el marco social dentro del cual se circunscribe su tarea educativa. Por ello, aspectos como la participación de las familias, la implicación de las autoridades locales y los organismos sociales, así como la flexibilidad en la organización escolar son aspectos fundamentales a considerar en una formación docente que busca una educación en la que todo el alumnado sea capaz de desarrollar las competencias esenciales que le permiten vivir y convivir plenamente (Ramírez, 2016).

Con base en lo anterior, se proponen algunos principios fundamentales en la formación inicial y continua del profesorado de educación básica dentro de una perspectiva comunitaria.

Propiciar el desarrollo individual y comunitario parte de competencias docentes colaborativas. La creación de las condiciones para que las personas logren su bienestar individual y social se fundamenta en procesos de formación docente que impulsan este desarrollo. La construcción de relaciones sociales cooperativas donde el intercambio, el convencimiento mutuo, el respeto a la diversidad y el reconocimiento a la participación son una constante se basa en un desempeńo docente reflexivo y compartido. Por ello, el trabajo colaborativo debe estar presente en todo el proceso de formación, así como la posibilidad de intervenir en los planes educativos y en el desarrollo de los proyectos que se están llevando a cabo en la comunidad. Una formación práctica basada en la reflexión crítica.

La formación docente involucra procesos de aprendizaje continuo. Al concebir una relación sistémica en la que todos los actores sociales y educativos colaboran conjuntamente en las decisiones que afectan al desarrollo de las personas y de su comunidad, los docentes se mantienen en una actitud receptiva y constructiva sobre las aportaciones que realizan otros agentes como la familia y las entidades sociales. En este sentido, los procesos de formación destacan el desarrollo del pensamiento flexible y crítico.

La orientación hacia un pensamiento autónomo y creativo. A partir de los vínculos que el profesorado genera con los agentes del entorno, este se configura como un agente que participa en las decisiones que impactan en su práctica profesional. En este sentido, el desarrollo de competencias, y la generación de ambientes abiertos que le permitan aportar desde sus conocimientos y experiencias son elementos sustanciales en los procesos de formación. De este modo, el desempeńo de sus funciones no se limita a una ejecución dentro del 
aula sino que se considera su relevancia como actor fundamental en el diseño y puesta en práctica de las propuestas educativas que se elaboran desde fuera del ámbito escolar.

La formación docente forma parte de la relación sistémica entre la escuela y la comunidad. El profesorado es un elemento de la comunidad y dentro de esta realiza sus funciones. Por ello, se asume que tanto su formación inicial como permanente están basadas en las necesidades sociales más relevantes. De este modo, en los espacios de formación es importante enfatizar la realización de prácticas en las que los docentes están en contacto con la comunidad y aprenden desde la atención a sus demandas. Así, en su preparación y mejora continua se incorporan los valores del desarrollo comunitario y el de la responsabilidad que se adquiere al propiciar la adquisición de competencias básicas para el desenvolvimiento social.

\section{Conclusiones}

Actualmente, la desigualdad a nivel mundial demanda propuestas educativas y sociales que contribuyan a reducir las brechas existentes entre las personas y las sociedades, las cuales impiden que una importante parte de la población en todo el planeta tenga acceso a las condiciones básicas para vivir plenamente. Un ejemplo de ello son los alarmantes niveles de exclusión social. A pesar de que se han hecho relevantes esfuerzos para disminuir esta condición, el Informe sobre Desarrollo Humano (2016) señala que una de cada nueve personas en el mundo padece hambre y una de cada tres, malnutrición. Asimismo, cada minuto 24 personas son desplazadas de manera forzada de sus hogares, siendo las minorías étnicas, las mujeres y las niñas quienes más padecen las consecuencias de esta situación. Sobre este punto, el informe acota que 370 millones de personas de 70 países han declarado sufrir discriminación en el acceso a la educación en su propio idioma, a los recursos naturales y al derecho de propiedad intelectual.

Ante este panorama, pensar en la educación con sentido comunitario permite des-mitificar que es inevitable la existencia de personas al margen del bienestar social. Asimismo, posibilita des-normalizar que la desigualdad en el ejercicio de derechos y en la obtención de oportunidades son un mal del que no se puede escapar en el logro de la estabilidad social, donde algunos alcanzan su desarrollo y otros ya lo conseguirán en algún momento. Esta forma de aceptar una realidad excluyente naturaliza la existencia de la marginación social como un daño colateral en las acciones humanas (Bauman, 2011), en las que existen víctimas fatales en un mundo donde unos ganan y otros pierden. 
Así, en el ámbito educativo algunos logran el éxito escolar y otros no, algunos desarrollan sus capacidades y otros no porque la condición humana es así, dentro de un ámbito de competitividad en donde algunos son considerados capaces y dignos de reconocimiento mientras que otras personas solo se quedan en un proceso cruel y excluyente. Bajo la lógica de este proceso, el profesor solo tiene que ejecutar ciertos lineamientos, emprender determinadas acciones y determinar quiénes son capaces para vivir y quiénes no lo conseguirán, esperando que tal vez en otro momento de su vida alancen los aprendizajes esperados, pero siendo una acción fuera de su alcance.

Pensar en una educación con sentido comunitario implica cambiar el paradigma de la función docente dentro de las sociedades. El planteamiento de proyectos educativos que parten de la reflexión, la flexibilidad, la participación en el establecimiento de acuerdos y la búsqueda del bienestar en todas las personas implica desmontar la idea de que los docentes son ejecutores de planes educativos y afirmar que son participantes de un planteamiento formativo que no es ajeno a ellos. Ubicar su desempeńo dentro de un entorno involucra acciones en los que su formación se fundamenta en la comunidad y en la que el contacto con el alumnado no se limita a lo que sucede en el aula sino que implica procesos de reconocimiento y comprensión de los diversos ambientes en los que se desenvuelven.

Por lo tanto, se trata de un proceso en el que al educar a otros se educa a sí mismo. A través de las interacciones que establece con agentes escolares y sociales profundiza el conocimiento que tiene sobre el alumnado en el aula y proporciona un sentido social a las funciones que desarrolla. De este modo, su labor no se limita a contribuir a la adquisición de ciertas competencias individuales sino que se extiende a la búsqueda del bien social mediante su desempeño.

La formación docente, desde este enfoque, fomenta la creación y el mantenimiento de profesionales de la educación comprometidos y conscientes de que su trabajo coadyuva a una mejora personal y social, que en sus manos tienen una importante labor para contribuir a terminar con situaciones de marginación y exclusión social. La necesidad de mirar a la comunidad en los procesos formativos se hace cada vez más urgente ante los ínfimos resultados que tienen los planes para aminorar las condiciones de pobreza y desigualdad, $\mathrm{y}$ ante las diferentes dimensiones que estas situaciones van adquiriendo. Remitirse a la comunidad es fortalecer procesos educativos que empoderan a las personas y crean profesionales de la educación con la seguridad y la capacidad para transformar tanto la realidad con la que trabajan como la de su propio desempeño. 
La educación con sentido comunitario: reflexiones en torno a la formación del profesora

\section{REFERENCIAS BIBLIOGRÁFICAS}

Bauman, Z. (2011). Daños colaterales. Desigualdades sociales en la era global. Buenos Aires, Argentina: FCE.

Bertalanffy, L. (1981). Teoría general de los sistemas: fundamentos, desarrollo y aplicaciones. Madrid, Espańa: Fondo de Cultura Económica.

Blanco, R. \& Duk, C. (2011). Educación inclusiva en América Latina y el Caribe. Aula, 17, 37-55.

Bueno, J. R. (1991). Hacia un modelo de Servicios Sociales de Acción Comunitaria. Madrid, España: Editorial Popular.

Bustos, A. (2011). Escuelas rurales y educación democrática. La oportunidad de la participación comunitaria. REIFOP, 14(2), 105-114.

Cardona, R. \& Ortells, M. (2012). ¿Caminamos hacia una educación inclusiva? Edetania, 41, 105-128.

Carneiro, R. (2012). Las TIC y los nuevos paradigmas educativos: la transformación de la escuela en una sociedad que se transforma. En R. Carneiro, J. C. Toscano y T. Díaz (eds.), Los desafios de las TIC para el cambio educativo (pp. 15-28). Madrid, España: OEI-Fundación Santillana.

Castro, M. L., Rodríguez, M. J. \& Urteaga, E. (2013). Abriendo las aulas: la vinculación entre docencia, investigación y cooperación comunitaria. Revista Electrónica Interuniversitaria de Formación del Profesorado, 16(3), 33-47. https://doi.org/10.6018/reifop.16.3.186741

Chieh, L. \& Vázquez, E. (2009). Schools consultans as agents of social justice for multicultural children and families. Journal of Educational and Psychological Consultation, 19(1), 26-44. https://doi.org/10.1080/10474410802462769

Cieza, J. A. (2006). Educación comunitaria. Revista de Educación, 339, 765-799.

Escudero, J. M. \& Martínez, B. (2011). Educación inclusiva y cambio escolar. Revista Iberoamericana de Educación, 55, enero-abril.

Gallego, C. (2010). El apoyo inclusivo desde la perspectiva comunitaria. Revista Interuniversitaria de Formación del Profesorado, 70(25,1), 93-109.

Gallego, C., Rodríguez, M. \& Corujo, C. (2016). La perspectiva comunitaria en la educación inclusiva. Prisma Social. Revista de Investigación Social, 16, 60-110.

Gutmann, A. (2004). Unity and diversity in democratic multicultural education: creative and destructive tensions. En J.A Banks (ed.), Diversity and Citizenship Education: Global Perspectives (pp. 71-96). San Francisco, CA: Jossey Bass.

Hidalgo, N., Siu, S. F., Bright, J., Swap, S. \& Epstein, J. (1995). Research on families, schools and communities: A multicultural perspective. En J. Banks y C. A. McGee-Banks (eds.), Handbook of Research on Multicultural Education (pp. 498-524). Nueva York, NY: Macmillan Publishing. 
Jaramillo, J. (2007). Las metáforas de lo comunitario. A propósito de una lectura crítica sobre el sentido de lo comunitario en la óptica de Zygmunt Bauman. Reflexión Política, 9(18), 20-31.

Johansen, P. \& Nielsen, N. (2012). Bridging between the regional degree and the community approaches to rurality. A suggestion for a definition of rurality for everyday use. Land Use Policy, 29, 781-788. https://doi.org/10.1016/ j.landusepol.2011.12.003

Lavaniegos, L. (2008). Competencias fundantes: sentido de auto apropiación y sentido comunitario. Xihmai, 3(5), 1-11.

Maya, I. (2004). Sentido de comunidad y potenciación comunitaria. Apuntes de Psicología, 22(2), 187-211.

Meza, M. (2011). El proyecto educativo integral comunitario: un instrumento para la promoción de la participación del docente. Investigación y Postgrado, 26(1), 129-154.

Morińa, A. \& Parrilla, A. (2006). Criterios para la formación permanente del profesorado en el marco de la educación inclusiva. Revista de Educación, 339, 517-539.

Neirotti, N. (2008). De la experiencia escolar a las politicas públicas. Proyectos locales de equidad educativa en cuatro paises de América Latina. Buenos Aires, Argentina: IIPE-UNESCO.

Perrenoud, P. (2005). El oficio de alumno y el sentido del trabajo escolar. Madrid, España: Editorial Popular.

Ramírez, A. (2016). Repensar la inclusión social desde la educación: algunas experiencias en América Latina. Revista Internacional de Educación para la Justicia Social, 5(1), 177-194.

Ramos, I., Holgado, D. \& Maya, I. (2014). El clima organizativo y el sentido de comunidad en la implicación comunitaria de las agrupaciones de las artes escénicas. Apuntes de Psicología, 32(2), 107-117.

Rodríguez, M. (2006).El asesoramiento comunitario y la reinvención del profesorado. Revista de Educación, 339, 59-75.

Simón, C., Giné, C. \& Echeita, G. (2016). Escuela, Familia y Comunidad: Construyendo alianzas para promover la inclusión. Revista Latinoamericana de Educación Inclusiva, 10(1), 25-42. https://doi.org/10.4067/ S0718-73782016000100003

Subirats, J. (2003). Participación y responsabilidades de la comunidad en la educación. Revista de Educación, 330, 217-236.

United Nations Educational, Scientific and Cultural Organization. (2005). Guidelines for inclusion: ensuring access to education for all. París: Unesco. 